\title{
Communication \\ BA.1 Omicron Variant of SARS-CoV-2: First Case Reported in Calabria Region, Italy
}

\author{
Cinzia Peronace ${ }^{1, *}$, Rossana Tallerico ${ }^{1}$, Manuela Colosimo ${ }^{1}$, Marco De Fazio ${ }^{1}$, Federica Pasceri ${ }^{1}$, Ilenia Talotta ${ }^{1}$, \\ Giuseppina Panduri ${ }^{1}$, Letizia Pintomalli ${ }^{2}$, Rosaria Oteri ${ }^{2}$, Valeria Calantoni ${ }^{2}$, Maria Teresa Fiorillo ${ }^{2}$, \\ Luca Gallelli $^{3}$, Erika Cione ${ }^{4, * \mathbb{D}}$ and Pasquale Minchella ${ }^{1}$
}

Citation: Peronace, C.; Tallerico, R.; Colosimo, M.; De Fazio, M.; Pasceri, F.; Talotta, I.; Panduri, G.; Pintomalli, L.; Oteri, R.; Calantoni, V.; et al. BA.1 Omicron Variant of SARS-CoV-2: First Case Reported in Calabria Region, Italy. COVID 2022, 2, 211-215. https://doi.org/10.3390/covid2030016

Academic Editor: Roger Frutos

Received: 21 January 2022

Accepted: 12 February 2022

Published: 22 February 2022

Publisher's Note: MDPI stays neutral with regard to jurisdictional claims in published maps and institutional affiliations.

Copyright: (C) 2022 by the authors. Licensee MDPI, Basel, Switzerland. This article is an open access article distributed under the terms and conditions of the Creative Commons Attribution (CC BY) license (https:// creativecommons.org/licenses/by/ $4.0 /)$.
1 Microbiology and Virology Unit, Pugliese-Ciaccio Hospital, 88100 Catanzaro, Italy; rossana.tallerico@gmail.com (R.T.); manuelacolosimo@hotmail.it (M.C.); marco_2592@yahoo.it (M.D.F.); federicapasceri@gmail.com (F.P.); ilenia.talotta@gmail.com (I.T.); pandurigiuseppina@gmail.com (G.P.); pminchella@aocz.it (P.M.)

2 Unit of Microbiology and Virology, North Health Center ASP 5, 89123 Reggio di Calabria, Italy; letizia.pintomalli@libero.it (L.P.); oterirosaria@gmail.com (R.O.); valeriacal@libero.it (V.C.); mariatfiorillo@hotmail.com (M.T.F.)

3 Department of Health Science, University of Catanzaro, Mater Domini Hospital, 88100 Catanzaro, Italy; gallelli@unicz.it

4 Department of Pharmacy, Health, and Nutritional Sciences, University of Calabria, 87036 Rende, Italy

* Correspondence: cinziaperonace@hotmail.it (C.P.); erika.cione@unical.it (E.C.); Tel.: +39-0961-883-202 (C.P.); +39-0844-93193 (E.C.)

\begin{abstract}
In December 2019, severe acute respiratory syndrome coronavirus 2 (SARS-CoV-2), the etiologic agent of coronavirus disease 2019 (COVID-19), emerged in Wuhan, China. Several variants of concern (VOCs) have been identified so far. Recently, the B.1.1.529 (Omicron) variant of SARSCoV-2 spread rapidly worldwide. We describe the first case of the Omicron genetic lineage BA.1 in our region. The patient is a physician who traveled to Johannesburg (South Africa) and returned to Reggio Calabria (Italy). He underwent a SARS-CoV-2 screening before leaving-a required procedure where travelers present a negative PCR test one-day prior to departing. Three days after arriving in Italy, he started experiencing cold symptoms. Clinically, he was without fever or severe respiratory symptoms and reported suffering from a cold and sore throat. The nasopharyngeal swab specimen was tested by TaqPath COVID-19 RT-PCR and sequenced by Sanger sequencing, and next-generation sequencing (NGS) data were processed with their relative software. A peculiar drop-off of the $S$ gene was obtained with TaqPath COVID-19 RT-PCR. S gene mutations indicative of the Omicron variant were obtained with both sequencing methods, pointing out 17 mutations in the 29 recognized by Sanger and the 28 recognized by NGS.
\end{abstract}

Keywords: Omicron; NGS; mutations

\section{Introduction}

In December 2019, severe acute respiratory syndrome coronavirus 2 (SARS-CoV-2), the etiologic agent of coronavirus disease 2019 (COVID-19), emerged in Wuhan, China. Several variants of concern (VOCs) have been identified so far. Recently, the discovery of the highly mutated Omicron variant of concern (VOC) G.R./484A (B.1.1.529) of SARS-CoV-2 was firstly detected in specimens collected on 11th November 2021, in Botswana and three days later (November 14th) in South Africa [1,2], which raised many questions. With the confirmed cases of Omicron reported in the European Union, there was an urgent need to trace it to monitor its spread [3]. This is of fundamental importance for setting up an effective countermeasure. More than half a million genome sequences were submitted to the Global Initiative on Sharing Avian Influenza Data (GISAID) from all six continents to confirm variants before December 2021, including this case from our laboratories the Department of Microbiology and Virology, Pugliese Ciaccio's Hospital, Catanzaro, Italy, 
and the Unit of Microbiology and Virology, North Health Center ASP 5, Reggio Calabria, Italy, respectively. At the time of writing this report, twenty-seven cases were reported to be the Omicron variant in Italy, of which one comes from the Calabria Region [4,5]. Besides that, a recent report indicates that two patients tested positive with Omicron on November 13 and 18 in Hong Kong, China [6]. It is believed that the first passenger transmitted the virus to the other passenger despite being in a hotel quarantine in different rooms [6,7]. Therefore, more studies are needed to better understand its transmissibility, immunity escape potential, clinical presentation and disease severity and the role of other available diagnostic and therapeutic countermeasures. Herein, we describe the first case of the Omicron genetic lineage BA.1 associated with mild clinical symptoms.

\section{Case Presentation Section}

On 5 December 2021, a 30-year-old man without comorbidities presented voluntarily to the hospital for a SARS-CoV-2 screening. He informed the hospital he had traveled to Johannesburg (South Africa) and back home to Reggio Calabria (Italy). In Johannesburg, he worked at a Trauma Unit as a physician for about one month. He had a SARS-CoV-2 screening before leaving - a procedure that requires travelers to present a negative PCR test one-day prior to departing. Three days after arriving in the Italy, he was started experiencing cold symptoms. Clinically, he was without fever or any severe respiratory symptoms. However, he was suffering from a cold and a sore throat. The patient had received two doses of the COVID-19 vaccine (BNT162b2) and received the booster dose on 6 November 2021. Therefore, blood specimens and nasopharyngeal swabs were collected. Markers of inflammation, including pro-calcitonin, C-reactive protein (CRP), natural killer cells and B and T lymphocytes, were immediately evaluated. The serological tests showed that he was positive for SARS-CoV-2-specific IgG. Unfortunately, the molecular nasopharyngeal swab resulted positive for SARS-CoV-2 RNA. Consequently, the patient was immediately notified.

\section{Methods}

Testing was carried out with the TaqPath ${ }^{\mathrm{TM}}$ COVID-19 CE-IVD RT-PCR Kit (hereinafter referred to as TaqPath ${ }^{\mathrm{TM}}$ COVID-19) (Thermo Fisher Scientific, Waltham, MA, USA), which targets the following genes: (i) open reading frame (ORF)1ab; (ii) nucleocapsid (N); and (iii) spike (S), coupled with QuantStudio 5 DX ThermoFisher Real-Time PCR (RT-PCR) (Thermo Fisher Scientific, Waltham, MA, USA). At the time of the study, the nasopharyngeal sample was collected in Universal Transport Media (UTM), opened in biosafety cabinet class-II and then $200 \mu \mathrm{L}$ of the UTM was further processed for viral nucleic acid extraction. Briefly, $200 \mu \mathrm{L}$ of UTM was extracted for the TaqPath ${ }^{\mathrm{TM}}$ COVID-19 (Thermo Fisher Scientific, Waltham, MA, USA). in the semi-automatic KingFisher Duo Prime by ThermoFisher using the MagMAX ${ }^{\mathrm{TM}}$ Viral/Pathogen Nucleic Acid isolation kit on the KingFisher Flex Purification system (Thermo Fisher Scientific, Waltham, MA, USA). Before RNA extraction, $10 \mu \mathrm{L}$ of Proteinase $\mathrm{K}$ was added to each well in the KingFisher ${ }^{\mathrm{TM}}$ Deep 96-well Plate (Thermo Fisher Scientific, Waltham, MA, USA). In addition, $10 \mu \mathrm{L}$ of the MS2 Phage Control, together with $10 \mu \mathrm{L}$ of magnetic beads, was added to all specimens. In addition, the specimen underwent genomic characterization following two methodologies: the Sanger-based sequencing by the SeqStudio Genetic Analyzer (Thermo Fisher Scientific, Waltham, MA, USA) and whole-genome based on next-generation sequencing (NGS) by MiSeq System (Illumina, San Diego, CA, USA). The latter was performed by the Regional Center at the Microbiology and Virology Laboratory of Catanzaro.

\section{Results and Discussion}

From the RT-PCR amplification curves, the shape highlights Ct values of 26.42 and 26.94 for ORF1ab and N genes, respectively, failing to identify the $S$ gene (data not shown). This peculiar drop-off of the $S$ gene was indicative of a possible variant of concern (VOC). Sequencing Sanger based on the S gene was performed using a validated, standard pro- 
tocol with 12 commercial primer pairings, as described by Paden et al. [7], for generating high-quality, full-length SARS-CoV-2 genomes from primary samples. The protocol uses multiplex reverse transcription PCR, followed by Sanger and MiSeq sequencing. Data analysis conducted on SeqScape software revealed the Omicron variant, lineage BA.1 (clade $21 \mathrm{k}$ ), recognizing 29 mutations. The NGS approach provided $2 \times 250 \mathrm{bp}$ read-length data. The SOPHIA DDM Platform analyzed the FASTQ reads. Clade analysis conducted on the ICOGEN Platform revealed 28 mutations in the $S$ gene, of which 17 were common to both systems, as shown in Table 1. As expected, the NGS data pointed out other mutations in the genome region of the virus: E T9I, M A63T, M D3G, M Q19E, N E31del, N G204R, N P13L, N R32del, N R203K, N S33del, NSP2 E563D, NSP3 A1892T, NSP3 K38R, NSP3 L1266I, NSP3 S1265del, NSP4 T492I, NSP5 P132H, NSP6 G107del, NSP6 I189V, NSP6 L105del, NSP6 S106del, NSP12 P323L and NSP14 I42V. Then, the lineage information was described using the Pangolin nomenclatures [7], and the Omicron variant sequences were deposited in the GISAID database on 9 December 2021. Consensus accuracy, including single-nucleotide polymorphisms and indels, is critical for determining coronavirus lineage and transmission networks. VOC are still integral to controlling virus spread. Therefore, a small aliquot of the collected specimen was processed through a rapid antigen test (AgRT) device based on a lateral-flow immuno-chromatographic assay (GeneFinder COVID-19 Ag Plus Rapid Test) (Osang Healthcare Co., Ltd, Daegu, Republic of Korea) [8]. The device showed positive results, indicating how an AgRT with high sensitivity and specificity represents an excellent screening method, especially in high prevalence areas. Additionally, as in this case, an AgRT can detect the Omicron variant. Further, nasopharyngeal swabs were collected to monitor the viral load up to a negative result, which was achieved in about ten days. From a clinical point of view, symptomatology did not worsen, which is in agreement with other clinical characteristics of the Omicron variant infection, which consisted, for the most part, of mild symptoms [9]. However, as with all variants, a lag exists between infection and more severe outcomes, and symptoms are expected to be milder in vaccinated persons and those with previous SARS-CoV-2 infection than in unvaccinated persons [10]. Our patient received two doses of the COVID-19 vaccine (BNT162b2) and received the booster dose on 6 November 2021. He was without fever or any severe respiratory symptoms. He was only suffering from a cold and a sore throat. Although mRNA vaccines provide protection mediated by T-cells, the response may still provide partial protection against mutations that allow the virus to escape from antibodies, significantly decreasing the severity and hospitalization rate, as in our case. With this evidence, we speculate that the immune responses elicited by vaccines could have alleviated the disease progression course, as reported in our case. The trials of vaccines performed in 2020-2021 clearly show the efficacy against SARS-CoV-2 infection. However, the vaccine effectiveness with the arrival of the new variant, Omicron, remains unclear. It is worth noting that the clinical features and epidemiologic data of the BA.1 Omicron infection are still being defined. This study, which included one patient who experienced reinfection after having been fully vaccinated, suggests also a shorter incubation period and a clinical condition associated with the other variant in persons who have been vaccinated, and the study adds to existing evidence of potential reinfection. The average incubation period has been described as $\geq 5$ days for SARS-CoV-2 and nearer to 4 for the B.1.617.2 (Delta) SARS-CoV-2 variant [10-12]. In this case, the average incubation period observed was about 3 days. Few clinical descriptions of Omicron infections are available, and mild illness amongst vaccinated subjects has been reported in California [13]. Although the vaccine produces a whole array of antibodies against the RBD-S spike protein, there are still many unknown mutations associated with the Omicron variant; therefore, partial immune escape may be expected. Lastly, more studies are needed to better understand the Omicron variant's transmissibility, clinical presentation, immunity escape potential and disease severity, as well as the role of other available diagnostic and therapeutic countermeasures. 
Table 1. Spike amino acid Omicron variation (ND = Not Detected).

\begin{tabular}{|c|c|c|}
\hline$\#$ & Sanger-Based Sequencing & Next-Generation Sequencing \\
\hline 1 & A67V & A67V \\
\hline 2 & T95I & T95I \\
\hline 3 & D215EPED & ND \\
\hline 4 & S371L & S371L \\
\hline 5 & S373P & S373P \\
\hline 6 & S375F & S375F \\
\hline 7 & K417N & K417N \\
\hline 8 & N440K & N440K \\
\hline 9 & G446S & G446S \\
\hline 10 & S477N & ND \\
\hline 11 & $\mathrm{~T} 478 \mathrm{~K}$ & ND \\
\hline 12 & $\mathrm{E} 484 \mathrm{~A}$ & ND \\
\hline 13 & Q493R & ND \\
\hline 14 & G496S & ND \\
\hline 15 & Q498R & ND \\
\hline 16 & N501Y & ND \\
\hline 17 & Y505H & ND \\
\hline 18 & T547K & T547K \\
\hline 19 & D614G & D614G \\
\hline 20 & H655Y & H655Y \\
\hline 21 & N679K & N679K \\
\hline 22 & P681H & ND \\
\hline 23 & N764K & N764K \\
\hline 24 & D796Y & ND \\
\hline 25 & N856K & N856K \\
\hline 26 & Q954H & Q954H \\
\hline 27 & N969K & N969K \\
\hline 28 & L981F & L981F \\
\hline 29 & D1146 & ND \\
\hline 30 & ND & G142D \\
\hline 31 & ND & G339D \\
\hline 32 & ND & L212I \\
\hline 33 & ND & P681H \\
\hline 34 & ND & V70del \\
\hline 35 & ND & V143del \\
\hline 36 & ND & Y144del \\
\hline 37 & ND & H69del \\
\hline 38 & ND & N211del \\
\hline 39 & ND & Y145del \\
\hline 40 & ND & ins214EPE \\
\hline
\end{tabular}

\section{Conclusions}

In light of the emergence of a new SARS-CoV-2 variant associated with increased transmissibility and partially immune-escape, the new Omicron variant of COVID-19 has been detected in more than 40 countries on five continents. Although the drop-off of the $S$ gene was recognized by the TaqPath COVID-19 RT-PCR kit assay, genomic characterization is crucial for identifying emerging viral variants and remains essential for determining the neutralization capacity against all variants, including Omicron. Full-genome sequencing is a critical tool in understanding emerging viruses. In fact, the initial sequencing of SARS-CoV-2 showed limited genetic variation before, which increased rapidly.

Author Contributions: Conceptualization, C.P. and P.M.; methodology, I.T., R.T., M.D.F., F.P., M.T.F., G.P. and C.P.; software E.C., L.P., L.G. and M.C.; software, C.P., R.T., R.O., V.C. and E.C.; writingoriginal draft preparation, C.P. and P.M.; writing-review and editing, E.C. and M.C.; supervision, P.M. All authors have read and agreed to the published version of the manuscript.

Funding: This research received no external funding. 
Institutional Review Board Statement: All the procedures were following the 1964 Helsinki Declaration and its later amendments. In any case the study did not involve humans or animals.

Informed Consent Statement: The study was conducted using residual specimens. According to the Italian health public law, this type of study did not require specific, informed consent and/or ethics committee approval.

Data Availability Statement: Data supporting the reported results can be found at the Department of Microbiology and Virology, Pugliese Ciaccio's Hospital, Catanzaro, Italy, and at the Unit of Microbiology and Virology, North Health Center ASP 5, Reggio Calabria, Italy.

Acknowledgments: We thank the MDHealthcare S.r.l., Via Nizzeri, 2295022 Aci Catena (CT), Italy, for its support in the fee journal payment.

Conflicts of Interest: The authors declare no conflict of interest.

\section{References}

1. World Health Organization. WHO Coronavirus (COVID-19) Dashboard. Available online: https://covid19.who.int/2 (accessed on 20 December 2021).

2. CDC. Science Brief: Omicron (B.1.1.529) Variant; US Department of Health and Human Services, CDC: Atlanta, GA, USA, 2021. Available online: https://www.cdc.gov/coronavirus/2019-ncov/science/science-briefs/scientific-brief-omicron-variant.html (accessed on 20 December 2021).

3. South African Doctor Who First Spotted the Covid Omicron Variant Says Symptoms Seem 'Mild' so Far. Available online: https: //www.cnbc.com/2021/11/29/omicron-covid-variant-symptoms-heres-what-we-know-so-far.html (accessed on 1 December 2021).

4. European Centre for Disease Prevention and Control. Available online: https://www.ecdc.europa.eu/en/news-events/ epidemiological-update-omicron-data-15-december (accessed on 20 December 2021).

5. IstitutoSuperiore di Sanità. Available online: https://www.epicentro.iss.it/coronavirus/sars-cov-2-monitoraggio-varianti (accessed on 20 December 2021).

6. Gu, H.; Krishnan, P.; Ng, D.Y.; Chang, L.D.; Liu, G.Y.; Cheng, S.S.; Hui, M.M.; Fan, M.C.; Wan, J.H.; Lau, L.H.; et al. Probable Transmission of SARS-CoV-2 Omicron Variant in Quarantine Hotel, Hong Kong, China, November 2021. Emerg. Infect. Dis. 2022, 28, 460-462. [CrossRef] [PubMed]

7. Paden, C.R.; Tao, Y.; Queen, K.; Zhang, J.; Li, Y.; Ueahara, A.; Tong, S. Rapid, sensitive, full-genome sequencing of severe acute respiratory syndrome coronavirus 2. Emerg. Infect. Dis. 2020, 26, 2401-2405. [CrossRef] [PubMed]

8. Rambaut, A.; Holmes, E.C.; O’Toole, A.; Hill, V.; McCrone, J.T.; Ruis, C.; du Plesis, L.; Pybus, O.G. A dynamic nomenclature proposal for SARS-CoV-2 lineages to assist genomic epidemiology. Nat. Microbiol. 2020, 5, 1403-1407. [CrossRef] [PubMed]

9. Ong, D.S.Y.; Claas, E.C.J.; Breijer, S.; Vaessen, N. Comparison of the GeneFinder ${ }^{\mathrm{TM}}$ COVID-19 Plus realamp kit on the sample-toresult Platform ELITeInGenius to the national reference method: An added value of $\mathrm{N}$ gene target detection? J. Clin. Virol. 2020, 132, 104632. [CrossRef] [PubMed]

10. World Health Organization (WHO). What You Need to Know about the New Omicron COVID-19 Variant. Available online: https:/ / www.euro.who.int/en/health-topics/health-emergencies/coronavirus-covid-19/news/news/2021/12/whatyou-need-to-know-about-the-new-omicron-covid-19-variant (accessed on 9 December 2021).

11. Torjesen, I. Covid-19: Omicron may be more transmissible than other variants and partly resistant to existing vaccines, scientists fear. BMJ 2021, 375, n2943. [CrossRef] [PubMed]

12. Pouwels, K.B.; Pritchard, E.; Matthews, P.C.; Stoesser, N.; Eyre, D.W.; Vihta, K.-D.; House, T.; Hay, J.; Bell, J.I.; Newton, J.N.; et al. Effect of Delta variant on viral burden and vaccine effectiveness against new SARS-CoV-2 infections in the UK. Nat. Med. 2021, 27, 2127-2135. [CrossRef] [PubMed]

13. Jansen, L.; Tegomoh, B.; Lange, K.; Showalter, K.; Figliomeni, J.; Abdalhamid, B.; Iwen, P.C.; Fauver, J.; Buss, B.; Donahue, M. Investigation of a SARS-CoV-2 B.1.1.529 (Omicron) Variant Cluster-Nebraska, November-December 2021. MMWR. Morb. Mortal. Wkly. Rep. 2021, 70, 1782-1784. [CrossRef] [PubMed] 